\title{
Soziokulturelle Gesundheitsdeterminanten gehen uns alle an!
}

Who will be the agents to bring the attention of policy makers for ... action on the determinants of health? Why not the medical profession? [1]

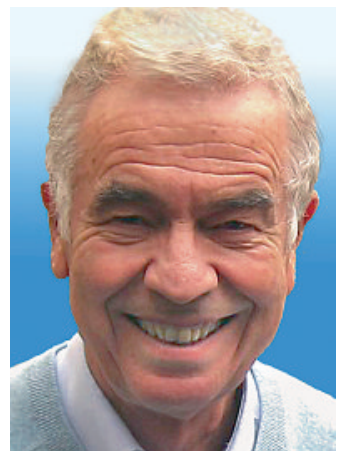

Hans Stalder
Vor 40 Jahren haben die Whitehall-Studien gezeigt, dass soziale Determinanten die Mortalität in gleichem oder sogar höherem Masse beeinflussen als alle anderen Risikofaktoren zusammen [2]. Nahezu zeitgleich wurde in der WHO-Erklärung von Alma Ata neben dem körperlichen und geistigen Wohlergehen das soziale ausdrücklich in die Definition des Gesundheitsbegriffs eingeschlossen. Die Beobachtungen aus England wurden weltweit bestätigt [3]: Je weiter die Schere des sozioökonomischen und/oder des Bildungsniveaus auseinanderklafft (und sie tut es immer mehr), desto grösser wird der Unterschied zwischen dem Gesundheitszustand von Arm und Reich. Arme stehen in fast jedem medizinischen Bereich schlechter da: mehr Krebs, mehr Gefässkrankheiten, mehr Alzheimer usw., und sie sterben mehrere Jahre früher. Gewiss häufen sich bei sozial Schwächeren auch die klassischen Risikofaktoren wie Alkohol und Rauchen, doch zum einen liegen dem wiederum die sozialen Faktoren zugrunde als Ursache der Ursache -, und zum anderen verbleiben immer noch rund $40 \%$, die allein auf soziokulturelle Faktoren zurückzuführen sind. Die Pathogenese ist derzeit noch ungeklärt. Wahrscheinlich üben psychosoziale Faktoren - Stress, innere Spannung, Kontrolle über das eigene Leben, Empowerment - ihre Wirkung über neuro-humorale Systeme aus.

Dieselben Beobachtungen sind auch für die Schweiz beschrieben. Das Risiko, bis zum 65. Lebensjahr zu sterben, ist bei Menschen, die körperlich arbeiten, um ein Viertel grösser als bei Intellektuellen (20\% gegenüber $15 \%)$, oder anders ausgedrückt: Mit jedem zusätzlichen Ausbildungsjahr nimmt die Lebenserwartung um $8 \%$ zu [4].

Wichtig dabei ist, dass diese Unterschiede graduell sind - die Ärmsten unterliegen höheren Risiken als weniger Arme, die Bestsituierten niedrigeren als Gutsituierte. Die gesundheitliche Disposition ist also nicht in zwei, sondern in viele Klassen unterteilt. Das darf jedoch kein Grund sein, ein Mehrklassen-Gesundheitssystem zu akzeptieren!

Die WHO hat vorgeschlagen - und verschiedene Länder, darunter Grossbritannien und Schweden, haben damit begonnen - Massnahmen zum Abbau dieser Differenzen zu ergreifen [5]. Die meisten sind nichtmedizinischer Art: Verbesserung der Lebensund Arbeitsbedingungen, schulische Förderung von Kindern aus benachteiligten Familien, Abbau sozialer Ungleichheit durch gerechte Steuern und nicht zu- letzt Erforschung der sozialen Einflüsse auf die Gesundheit durch gezielte Studien.

Ähnliche Vorschläge [6] sind bei uns auf wenig Echo gestossen, obwohl sie potentiell wirksamer wären als andere Massnahmen, wie z.B. die Erkennung und Behandlung von Risikofaktoren. Der Grund ist vermutlich die Tatsache, dass die Schweizer Politik des Gesundheitswesens - und übrigens meist auch die der medizinische Forschung - streng vom sozialen Sektor getrennt ist. Eine wirksame Gesundheitsförderung muss jedoch übergreifend ansetzen.

Selbst wenn der Zugang zum Gesundheitssystem nicht für jedermann der wichtigste Aspekt zu sein scheint, sollte die Politik ihn zumindest nicht noch erschweren. Genau das findet jedoch bei uns zunehmend statt: Die private Beteiligung an den Gesundheitsausgaben ist die höchste in der OECD; die Franchisen begünstigen Gesunde und Reiche, um die sich die Kassen ohnehin reissen; die Einführung von Spitalpauschalen und die Bemühungen, die Behandlung auf der Notfallstation einzudämmen, drohen Ärmere zusätzlich zu benachteiligen, weil das Spital für sie oft der einzige Ort ist, an dem sie eine angemessene Behandlung erhalten. Der Vorschlag einer Praxisgebühr wurde vom Parlament glücklicherweise abgelehnt, aber wie konnte das BAG überhaupt auch nur daran denken?

Was haben wir Ärzte mit alldem zu tun? Haben wir daran gedacht, vorbeugende Massnahmen vor allem bei sozial Schwächeren durchzuführen (statt Check-ups bei denen, die sie nicht brauchen ...), da doch hinreichend bekannt ist, dass Prävention umso mehr nützt, je höher das Risiko ist? Allerdings werden soziale Risikofaktoren von unseren Taschenrechnern nicht erfasst, und weder die sozialwissenschaftliche noch die medizinische Fakultät hat uns gelehrt, eine Sozialanamnese durchzuführen [7]. Sollten Mediziner und Sozialwissenschaftler nicht auch gemeinsam forschen, um die Bedeutung sozialer Faktoren für die Gesundheit besser zu erkennen? Und schliesslich kann sich jeder von uns einsetzen, auf Gemeinde-, Kantons- oder Bundesebene Massnahmen zu unterstützen, die dazu beitragen, das soziale Gefälle zu verringern, und solche zu verhindern, die dieses verstärken.

Ja, die soziokulturellen Gesundheitsdeterminanten gehen uns alle an!

Hans Stalder * 


\section{Literatur}

1 Marmot MG. Status syndrome: a challenge to medicine. JAMA. 2006;295(11):1304-7.

2 Marmot MG, Rose G, Shipley M, Hamilton PJ. Employment grade and coronary heart disease in British civil servants. J Epidemiol Community Health. 1978;32(4):244-9.

3 Hemingway H, Shipley M, Brunner E, Britton A, Malik M, Marmot M. Does autonomic function link social position to coronary risk? The Whitehall II study. Circulation. 2005;111(23):3071-7.

4 Bopp M, Minder CE. Mortality by education in German speaking Switzerland, 1990-1997: results from the Swiss National Cohort. Int J Epidemiol. 2003;32(3):346-54.
5 Closing the gap in a generation: health equity through action on the social determinants of health. Final Report of the Commission on Social. Determinants of Health. Geneva : World Health Organization; 2008 .

6 Lignes directrices pour une politique multisectorielle de la santé. OFSP ; 2005.

7 Wolff H, Stalder H, Morabia A. De la recherche à la pratique: la place de l'anamnèse sociale en médecine de premier recours. Med Hyg. 2004;62:1837-9. 